\title{
Influencia de la Localización del Almidón Mativo de Yuca en el Canal de Sedimentación sobre la Calidad del Almidón Agrio
}

\author{
Irene Tischer* \\ Martín A. Moreno**
}

* Ph. D. Profesora Titular de la Escuela de Ingeniería de Sistemas y Computación - Facultad de Ingeniería Universidad del Valle, Santiago de Cali, Colombia. e-mail: irene@eisc.univalle.edu.co

** MSc., Candidato a Doctor, Universidad Politécnica de Valencia España. Profesor Titular Escuela de Ingeniería Mecánica - Facultad de Ingeniería - Universidad del Valle, Santiago de Cali, Colombia.

e-mail:mamoreno@univalle.edu.co

Fecha de recepción: Abril 30 de 2004

Fecha de aprobación: Septiembre 6 de 2004

\section{RESUMEN}

El proceso productivo del almidón de yuca realizado artesanalmente en el Morte del Departamento del Cauca, incluye una etapa de sedimentación que actualmente se lleva a cabo en canales. Entre los procesadores de yuca de la región existe la opinión, que del inicio del canal se obtiene almidón agrio con un poder de expansión más alto, y por lo tanto se podría vender a un mejor precio.

En la presente investigación se analiza si existe una estratificación de calidades de acuerdo a la posición del almidón en el canal. Se evaluaron dos estrategias de recoger el almidón de los canales, a lo largo o perpendicular al canal. Los resultados 
obtenidos son negativos. Minguna de las estrategias permite una estratificación de calidades tan significante para que se logre obtener almidones que respondan a las exigencias de los diferentes mercados. Si se busca una calidad homogénea, se recomienda recoger el almidón perpendicular al canal.

Palabras clave: Agroindustria rural, almidón agrio, yuca, poder de expansión, canales de sedimentación.

\section{ABSTRACT}

The productive process of cassava starch made by artisans in the Morth of the Cauca Department includes a sedimentation stage that at the moment is carried out in channels. Among the processors of cassava of the region, the opinion exists that the starch taken from the beginning of the channel has a higher power of expansion, and therefore it would be possible to be sold at a better price.

In the present investigation it is analyzed if a stratification of qualities according to the position of the starch in the channel exists. Two strategies were evaluated to gather the starch of the channels, along or perpendicular to the channel. The obtained results are negative. Mone of the strategies allows a so significant stratification of qualities so that it is managed to obtain starches that respond to the requirements of the different markets. If a homogenous starch quality is desired, it is recommended to gather the starch perpendicular to the channel.

Key words: Rural agroindustry, sour starch, cassava, specific volume, sedimentation channels.

\section{INTRODUCCIÓN}

El proceso de extracción de almidón de yuca por vía húmeda se inicia con la desintegración de las raíces para romper la membrana de las células y permitir la liberación de los gránulos de almidón que se ubican dentro de ellas. Por el lavado y tamizado de la pulpa de yuca se separa la fibra de la "lechada", término como los procesadores definen la mezcla de agua con los gránulos de almidón, los solubles y las fibras finas que atraviesan el tamiz. La lechada es conducida mediante tuberías hacia tanques o canales donde el almidón se sedimenta debido a su mayor densidad respecto a la del agua. almidón sedimentado se recoge y deposita en tanques para lograr una fermentación natural anaerobia por un periodo de 15 a 60 días. El almidón fermentado se seca al sol, proceso por el cual el producto adquiere su poder de expansión o capacidad de hacer crecer una masa horneada, propiedad deseada por los consumidores del almidón agrio [RI+01].

En las plantas de proceso más tradicionales se utilizan tanques para la operación de la sedimentación; la lechada proveniente de los tamices se vierte en uno de ellos y cuando éste es llenado con la suspensión, se pasa al siguiente y así sucesivamente hasta completar los tanques disponibles. Estos tanques son desocupados el día siguiente quedando en el fondo el almidón precipitado formando una capa bien compacta. Sobre esta capa de almidón (con una densidad promedia de $1.63 \mathrm{~g} / \mathrm{cm}^{3}$ ) se depositan el material proteico de menor densidad (1.13 $\mathrm{g} / \mathrm{cm}^{3}$ ) que el almidón y las fibras finas (1.31 $\mathrm{g} / \mathrm{cm}^{3}$ ) que por no compactar de la misma forma que el almidón, pueden ser fácilmente retirados con agua [PIM80]. El problema de los tanques es que limita la capacidad de proceso de la planta debido a que el tiempo de retención de la lechada en el tanque es alto.

Desde la última década se están utilizando los canales de sedimentación, tecnología originaria de Europa y de amplio uso en Brasil. Los canales generalmente tienen unas dimensiones de 0.4 a $0.6 \mathrm{~m}$ de ancho, $0.4 \mathrm{~m}$ de profundidad, de 100 a $150 \mathrm{~m}$ de longitud y de pendiente 0 . La longitud del canal se logra construyendo 5 o 6 
tramos paralelos unidos en uno de sus extremos como se indica en la figura 1.

El principio de sedimentación en los canales consiste en una precipitación selectiva de la suspensión del almidón en movimiento. En un extremo del canal se recibe la lechada y durante el recorrido a lo largo de los tramos, los gránulos de almidón se van sedimentando mientras que las partículas livianas y compuestos en solución continúan con la corriente saliendo en el otro extremo con las aguas residuales [RI+01]. Según [TKA59] es importante controlar la velocidad de la corriente que debe ser mayor de $4 \mathrm{~m} / \mathrm{min}$ para evitar la sedimentación de las partículas finas y menor de $10 \mathrm{~m} / \mathrm{min}$ para asegurar flujo laminar y evitar pérdidas de almidón en las aguas residuales.

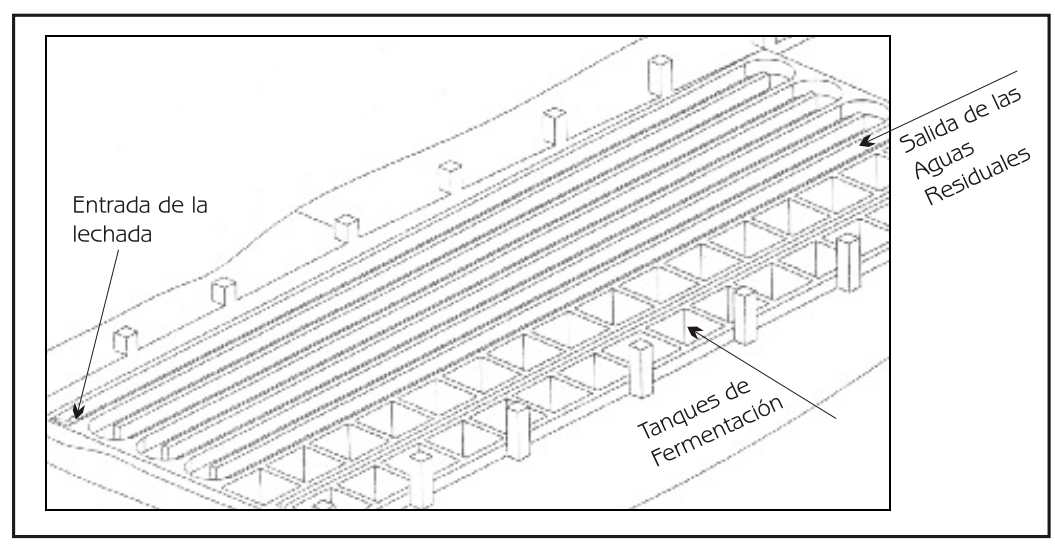

Figura 1. Los canales de sedimentación

La operación de sedimentación se realiza en forma continua durante varios días hasta copar la capacidad de los canales. El almidón de los canales, se retira en forma de bloques compactos que se depositan en los tanques de fermentación localizados a lo largo de los canales. Los procesadores recogen los bloques perpendicularmente a la dirección de los canales, buscando homogeneizar posibles variaciones de calidad, ya que creen que del primer canal se obtiene almidón agrio con un mejor poder de expansión. Utilizando los canales de sedimentación se ha logrado recuperar más almidón y de mejor calidad aumentando la eficiencia del proceso.

El modelamiento de la sedimentación de los gránulos de almidón como ocurre en tanques y canales de sedimentación, es complejo. Cuando una partícula sedimenta libremente está sujeta a la acción de tres fuerzas: la fuerza gravitacional en sentido del movimiento de la partícula, la fuerza de empuje y la fuerza de arrastre en dirección contraria. La partícula acelera hasta el punto en el que la fuerza de su peso se equilibra con la de empuje y arrastre $y$ entonces continúa el descenso a velocidad constante. Esta velocidad se define como la velocidad de sedimentación o velocidad terminal de sedimentación.

La Ley de Stokes permite determinar la velocidad de sedimentación para partículas esféricas menores de 100 micras si el flujo es laminar y si el fluido tiene una concentración de sólidos inferior al 5\%:

$$
v_{0}=g \cdot D_{p}^{2}\left(\rho_{p}-\rho_{f}\right) / 18 \mu
$$

Donde:

$v_{0}$ :velocidad de la partícula, $\mathrm{m} / \mathrm{s}$

$g$ :Aceleración de la gravedad, 9.8 $\mathrm{m} / \mathrm{s}^{2}$

$D_{p}$ : diámetro de la partícula, $\mathrm{m}$

$\rho_{p}$ : densidad de la partícula, $\mathrm{kg} / \mathrm{m}^{3}$

$\rho_{f}$ : densidad del fluido, $\mathrm{kg} / \mathrm{m}^{3}$

$\mu \quad$ :Viscosidad del fluido, $\mathrm{kg} /(\mathrm{m} \mathrm{s})$.

La velocidad de sedimentación de las partículas de acuerdo a la Ley de Stokes es directamente proporcional al cuadrado del diámetro de las partículas y como la velocidad también es igual al recorrido vertical de la partícula sobre el tiempo de caída utilizado, se puede concluir que este tiempo es proporcional al recorrido vertical de la partícula e inversamente proporcional al diámetro cuadrado de las mismas [PIM80].

La determinación de la velocidad de sedimentación de las partículas se vuelve más 
incierta a medida que aumenta la concentración de la solución debido a que los gradientes de velocidad alrededor de las partículas son perturbados por la presencia de otras partículas. Según [TKA59], cuando el contenido de sólidos es mayor del 5\%, la velocidad de sedimentación disminuye debido a la interferencia que hay por el choque de unas partículas con otras y se deben utilizar factores de corrección que indican que la velocidad de sedimentación disminuye en un $40 \%$ para suspensiones del 12 al $14 \%$ y en un $55 \%$ para suspensiones con concentraciones del $22 \%$.

Aún, asumiendo flujo laminar y partículas esféricas, es difícil calcular con precisión la velocidad de sedimentación de las partículas de almidón en la lechada debido a que los parámetros que la Ley de Stokes considera constantes, son muy variables en el proceso tradicional de extracción de almidón de yuca en el Cauca:

$\square$ El tamaño de las partículas que varía entre 5 y 35 micras, depende de factores poco controlables como la variedad y edad de las raíces de yuca, factores climáticos y época de cosecha.

च La densidad de los gránulos de almidón puede variar debido a que el almidón es un material biológico que sufre permanentes transformaciones de acuerdo a las necesidades de la planta.

■ La concentración de la lechada varía desde un $7.3 \%$ al inicio del tamizado hasta casi cero a los 15 ó 20 minutos que dura la operación.

$\square$ La velocidad de la corriente en el canal es variable ya que el almidón que va sedimentando cambia continuamente la pendiente.

Bajo los supuestos de la Ley de Stokes, los gránulos de mayor diámetro deberían sedimentarse al inicio del canal, hipótesis que sustenta la posibilidad de una estratificación del canal respecto a la calidad del almidón.
En [TI+99, LE+98] se muestra un análisis preliminar de la relación entre el poder de expansión respecto del tramo de canal donde ocurrió la sedimentación y de su composición granulométrica. El trabajo pretendía probar la hipótesis empírica de los procesadores sobre el mejor poder de expansión de los almidones sedimentados en los primeros tramos del canal. De ser positiva la hipótesis se podría utilizar el canal de sedimentación para separar antes de la fermentación almidones de diferentes calidades. Se obtuvieron los siguientes resultados: El canal de sedimentación trabaja como un clasificador por tamaño de los granos de almidón. Los granos más grandes predominan en los primeros metros del canal y los pequeños se ubican en mayor proporción al final del mismo. Mo se pudo establecer una relación clara entre la posición del almidón en el canal y su calidad. La presente investigación se centra en esta relación, buscando profundizar los anteriores resultados.

\section{OBJETIVOS}

En la presente investigación se analiza la influencia de la localización del almidón nativo en el canal de sedimentación sobre la calidad del almidón agrio. Se quiere determinar si existe una estratificación del poder de expansión de acuerdo a la posición del almidón en el canal.

Los objetivos específicos buscan evaluar estrategias que aprovechan de una posible estratificación de la calidad del almidón, para separar el almidón por calidad en los tanques de fermentación:

1. Analizar el poder de expansión del almidón sedimentado a lo largo de los canales de sedimentación.

2. Analizar el poder de expansión del almidón sedimentado recogido perpendicular a la dirección de los canales de sedimentación. 


\section{METODOLOGÍA}

\subsection{La planta de proceso:}

Los experimentos se realizaron en la Rallandería La Agustina localizada en la Vereda La Agustina, Corregimiento de Mondomo, Municipio de Santander de Quilichao. Predomina el clima medio con una temperatura de $22^{\circ} \mathrm{C}$ y una precipitación promedia de 2000 mm/año.

\subsection{La medición del poder de expansión:}

El método utilizado para determinar el poder de expansión, que constituye el principal criterio de evaluación de su calidad, consiste en el horneado a $2623^{\circ} \mathrm{C}$ por 22 minutos de una suspensión de $10 \mathrm{~g}$ de almidón agrio y $12 \mathrm{~g}$ de agua. Al producto resultante se le determina el peso y el volumen siendo la relación volumen a peso la medida cuantitativa final de capacidad expansiva del almidón.

Almidones agrios con un poder de expansión superior a $12 \mathrm{~cm}^{3} / \mathrm{g}$ se clasifican como extras, de $10-12 \mathrm{~cm}^{3} / \mathrm{g}$ como bueno, de $8-10 \mathrm{~cm}^{3} / \mathrm{g}$ como corriente y menores de $8 \mathrm{~cm}^{3} / \mathrm{g}$ como malos, a pesar de que se utilizan como ingrediente en la elaboración de algunos productos fritos [FE+99].

\subsection{Materiales:}

El material utilizado en los experimentos es el siguiente:

v Infraestructura y equipos de la Planta Piloto para el pesaje, lavado y rallado de las raíces, tamizado de la pulpa, tamizado de la lechada, sedimentación y secado del almidón.

$\square$ Tubos de PVC de 4 pg. de diámetro y de 1.20 $m$ de longitud, para simular la condición anaerobia de los tanques de fermentación.

$\checkmark$ Raíces de yuca algodona, parra y amarga.

■ Horno eléctrico para la prueba de expansión.

$\checkmark$ Horno eléctrico para determinar la humedad de las muestras.

■ Bolsas plásticas para el almacenamiento de las muestras.

च Laboratorio de análisis de alimentos de la Universidad del Valle, para realizar las pruebas de expansión del almidón agrio.

\subsection{Montaje de los experimentos:}

El experimento se realizó de la siguiente manera:

च Se seleccionaron los puntos para tomar las muestras a lo largo del canal. Se trabajó con los tres primeros tramos del canal. En cada tramo se seleccionaron 4 puntos para recoger el almidón sedimentado (ver figura 2).

$\square$ Se procesaron las raíces de la manera habitual hasta obtener suficiente almidón sedimentado en el canal, para poder sacar las muestras requeridas de los tramos 1 a 3 . El caudal de la lechada se mantuvo durante todo el ensayo en $1.2 \mathrm{l} / \mathrm{s}$.

च El almidón de cada punto se colocó en 2 tubos de PVC para una fermentación durante 30 días aproximadamente.

$\square$ Todas las muestras fermentadas se secaron al sol en el mismo día.

च Se evaluó el poder de expansión de cada tubo, aplicando el método descrito en 3.2.

च Se determinó el poder de expansión del almidón en un punto del canal, promediando los valores de los 2 tubos correspondientes.

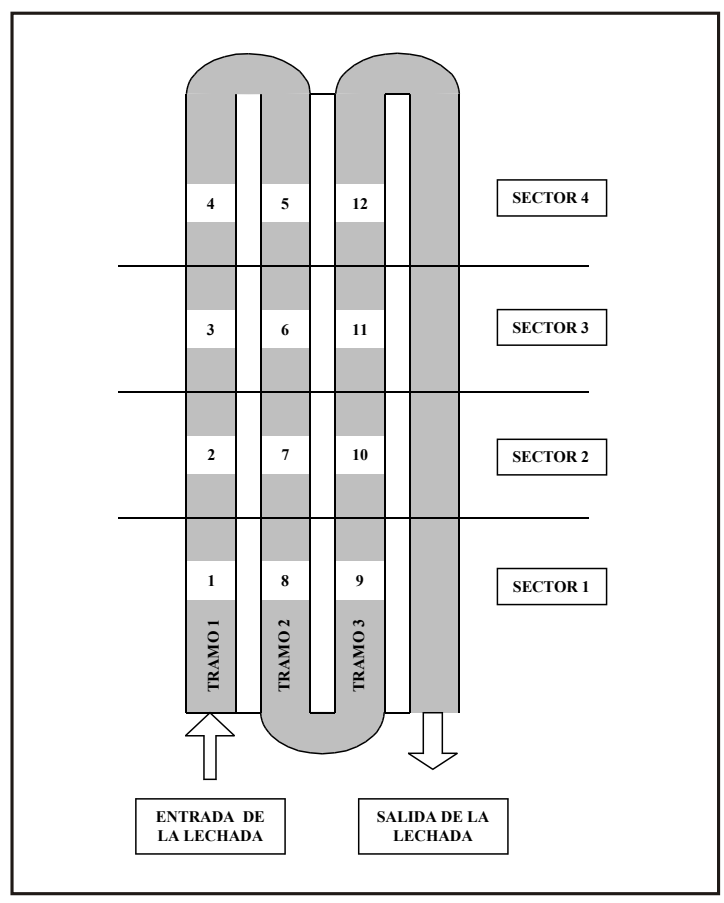

Figura 2. Diseño del experimento: los puntos 1-12, los 3 tramos y los 4 sectores. 


\subsection{Diseño estadístico:}

Para mostrar el efecto de la distancia en el canal sobre el Volumen de Expansión se utilizó un Modelo de Bloques Completos al Azar. Los bloques los conformaron las réplicas del experimento. Se eligió un modelo de Bloques porque las réplicas del experimento no se pudieron montar el mismo día, por limitaciones en la cantidad de material experimental: número de canales de sedimentación, cantidad de yuca requerida para el proceso. En el montaje del experimento no se controló la variedad de yuca ni la cantidad de la misma.

Se evaluaron las dos estrategias de recoger el almidón del canal:

Estrategia 1: se recoge el almidón por tramos del canal. Los poderes de expansión en los 4 puntos de cada tramo se consideran muestras del poder de expansión del tramo.

Estrategia 2: se recoge el almidón en 4 sectores que atraviesan el canal perpendicularmente. Así cada sector contiene 3 puntos, que se utilizan como muestras del poder de expansión del sector (figura 2).

El modelo estadístico para evaluar el efecto del canal sobre el poder de expansión, se puede expresar como:

$Y_{i j k}=\mu+\beta_{i}+p_{j}+\gamma_{k(i j)}+\varepsilon_{i j}$

$i \quad$ :identifica la réplica del experimento (bloque)

$j$ : se refiere a la posición en el canal: de acuerdo con la estrategia a evaluar el tramo o el sector analizado

$k$ :identifica la $k$-ésima muestra en la posiciónj

$Y_{i j k}$ :es el poder de expansión observado, proveniente de la réplica $i$, la posición $j$ y la muestrak

$\mu$ :es el promedio general del poder de expansión

$\beta_{i}$ :es el efecto de la i-ésima réplica sobre el poder de expansión

$p_{j}$ :es el efecto de la posición j sobre el poder de expansión

$\gamma_{k(i j)}$ :Es la interacción entre la i-ésima réplica del experimento y la posición j en la observación $k$

$\varepsilon_{i j} \quad$ es el error aleatorio correspondiente al valor observado de poder de expansión de la iésima réplica en la posiciónj-ésima.

\section{RESULTADOS Y DISCUSIÓN}

\subsection{Análisis global:}

La hipótesis de los procesadores y la ley de Stokes junto con el supuesto que los gránulos grandes producen un mejor almidón, sugieren que la expansión del almidón se reduce a lo largo del canal. Como muestra la figura 3, éste no es el caso. El poder de expansión obtenido a lo largo del canal varía mucho en forma no sistemática. Un análisis de varianzas muestra que el poder de expansión no tiene relación significativa (nivel de significancia 0.33) con la posición en el canal (punto).

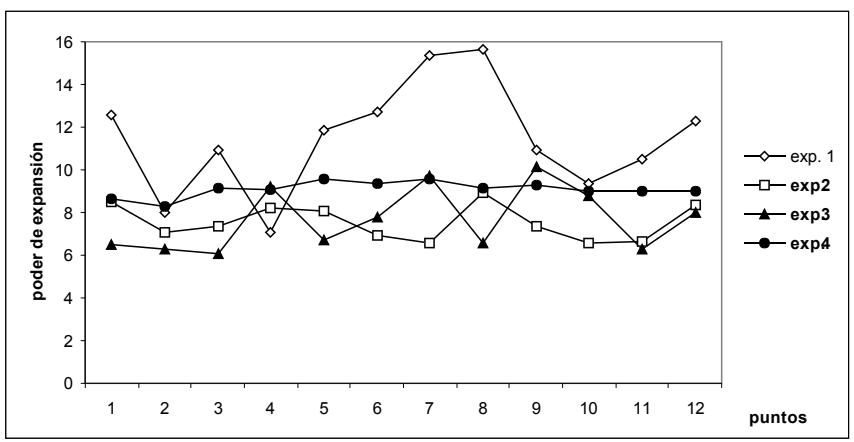

Figura 3. Poder de expansión del almidón a lo largo del canal

\subsection{Estrategia 1: Recoger el almidón por tramos del canal:}

El poder de expansión por tramo se muestra en la figura 4. Se grafica el promedio de los puntos de cada tramo. En forma general el tramo 2 presentó el mayor Volumen de Expansión (9.66), seguido por el tramo 3 (8.85) y el tramo 1 (8.31). El coeficiente de variación entre estos valores es muy bajo, es decir, no hay mucha diferencia entre los tramos. 


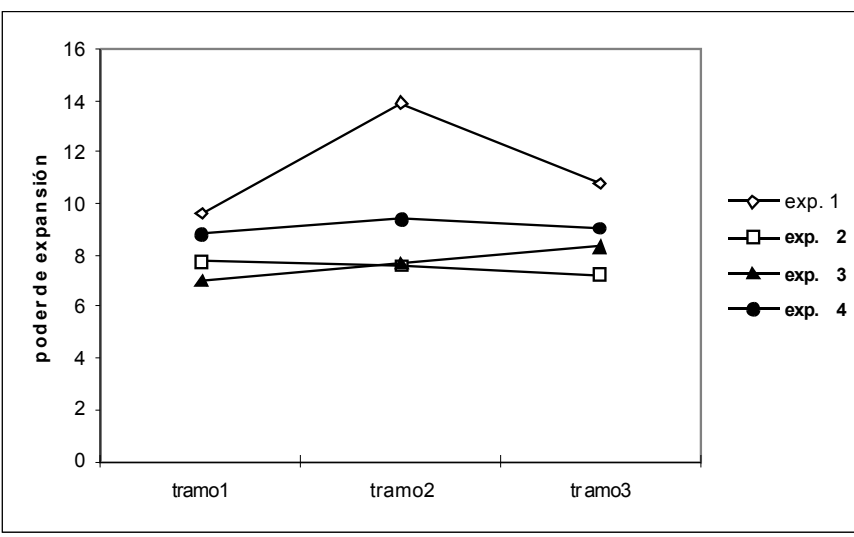

Figura 4. Poder de expansión del almidón en función de los tramos del canal.

La tabla resume los resultados del análisis de varianzas.

\begin{tabular}{|l|c|c|c|c|c|c|}
\hline Fuente de variación & $S S$ & $g l$ & $M S$ & $F$ & $P$ & $\begin{array}{c}F_{\text {crit }} \\
(\alpha=0.05)\end{array}$ \\
\hline Tramo & 14.66318 & 2 & 7.33159 & 4.200626 & 0.022926 & 3.259444 \\
\hline Experimento & 118.0385 & 3 & 39.34617 & 22.54335 & $2.17 \mathrm{E}-08$ & 2.866265 \\
\hline Interacción & 28.54922 & 6 & 4.758203 & 2.726208 & 0.027509 & 2.363748 \\
\hline Dentro de grupos & 62.83283 & 36 & 1.745356 & & & \\
\hline Total & 224.0837 & 47 & & & & \\
\hline
\end{tabular}

El análisis estadístico muestra que el tramo del canal afecta la calidad del almidón agrio (nivel de significancia de 0.023) y que hay interacción entre tramos y experimentos (nivel de significancia de 0.027). El tramo 2 tiene un mejor poder de expansión que los demás.

Analizando con más detalle los datos se aclara que este resultado se debe exclusivamente a la influencia del experimento 1 , en donde el poder de expansión muestra un comportamiento completamente diferente. En este experimento predominaron las raíces de la variedad algodona, reconocida en muchos estudios [DU+95, ZA+96] por la alta calidad de sus almidones. Posiblemente esta variedad se comporta distinto en cuanto a la sedimentación. En el análisis de varianzas sin el experimento 1 no se detecta una diferencia significativa entre los tramos.

La tabla siguiente resume los resultados del análisis de varianzas de los experimentos 2,3 y 4.

\begin{tabular}{|c|c|c|c|c|c|c|}
\hline Fuente de variación & $S S$ & $g l$ & $M S$ & $F$ & $P$ & $\begin{array}{c}F_{\text {crit }} \\
(\alpha=0.05)\end{array}$ \\
\hline Tramo & 1,050906 & 2 & 0,525453 & 0,501087 & 0,611396 & 3,354131 \\
\hline Experimento & 17,95517 & 2 & 8,977586 & 8,561278 & 0,001320 & 3,354131 \\
\hline Interacción & 3,735628 & 4 & 0,933907 & 0,8906 & 0,482968 & 2,727766 \\
\hline Dentro de grupos & 28,31293 & 27 & 1,048627 & & & \\
\hline Total & 51,05463 & 35 & & & & \\
\hline
\end{tabular}

\subsection{Estrategia 2: Recoger el almidón perpendicular a la dirección del canal:}

La figura 5 muestra el poder de expansión del almidón recogido por sectores. Se usó como valor de un sector el promedio de los 3 puntos correspondientes. Se observa que casi no hay diferencia del poder de expansión en los distintos sectores, con excepción del experimento 1, que en este ensayo resultó siempre distinto de los demás. Sin embargo, almidones con expansiones entre 10 y $12 \mathrm{~cm}^{3} / \mathrm{g}$ se clasifican en el grupo de los buenos, es decir, desde el punto de vista económico no se justificaría ni siquiera en el caso del experimento 1 aplicar la estrategia de la recolección por sectores para separar almidones de calidad superior.

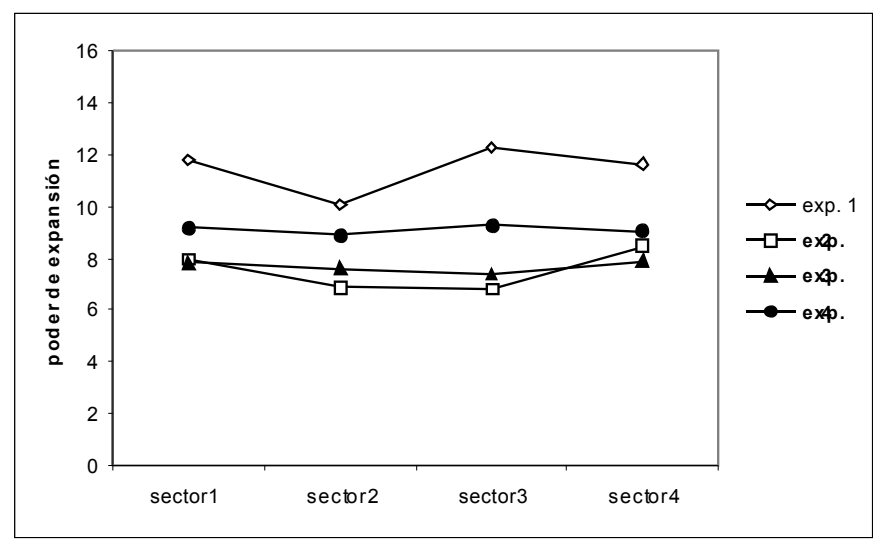

Figura 5. Poder de expansión del almidón en función de los sectores del canal.

El análisis estadístico confirma que no hay diferencia entre el poder de expansión de almidones provenientes de los diferentes sectores del canal. La variación entre sectores no es significativa. Se obtienen las expansiones promedias muy similares de $9.18,8.35,8.93 \mathrm{y}$ 9.28 . 


\begin{tabular}{|c|c|c|c|c|c|c|}
\hline Fuente de variación & $S S$ & $g l$ & $M S$ & $F$ & $P$ & $\begin{array}{c}F_{\text {crit }} \\
(\alpha=0.05)\end{array}$ \\
\hline Sector & 6.218573 & 3 & 2.072858 & 0.730373 & 0.541491 & 2.901118 \\
\hline Experimento & 118.0385 & 3 & 39.34617 & 13.86366 & $5.8 \mathrm{E}-06$ & 2.901118 \\
\hline Interacción & 9.008119 & 9 & 1.000902 & 0.352669 & 0.948979 & 2.188763 \\
\hline Dentro de grupos & 90.81853 & 32 & 2.838079 & & & \\
\hline Total & 224.0837 & 47 & & & & \\
\hline
\end{tabular}

\section{CONCLUSIONES Y RECOMENDACIONES}

\section{Análisis global}

Mo se encontró una relación contundente entre el poder de expansión y los puntos del canal. Como regla general se concluye que si la yuca es de calidad, todo el almidón obtenido es de calidad y viceversa.

\section{Estrategia 1: Recoger el almidón por tramos del canal}

Los tramos del canal estratifican la calidad del almidón resultante. Sin embargo, las diferencias en los valores del poder de expansión del almidón proveniente de los diferentes tramos son muy pequeñas y en la práctica no representan beneficios económicos.

En uno de los experimentos se encontró una estratificación de las calidades, en los otros resultaron calidades iguales en todos los tramos. Por ende no se puede predecir si habrá o no una estratificación por tramo. En conclusión: no es recomendable la estrategia 1 ni para estratificar ni para homogenizar las calidades del almidón.

\section{Estrategia 2: Recoger el almidón perpendicular a la dirección del canal}

Mo se da la estratificación por calidad del almidón recogido por sectores. Sin embargo, aplicando esta estrategia se obtienen calidades de almidón muy homogéneas. Es conveniente recomendar esta práctica a los procesadores con el fin de normalizar su producto.

\section{Recomendaciones}

Se recomienda realizar experimentos de estratificación exclusivamente con la variedad algodona, dado que ésta predominó en el experimento donde se obtuvo una separación de calidad por tramos.

Dado que el proceso de sedimentación depende cuantitativa y cualitativamente de las dimensiones del canal, se recomienda repetir experimentos similares en otras plantas.

\section{REFERENCIAS}

[DU+95] DUFOUR D., BRABET C., ZAKHIA M., CHUZEL G., 1995. Influence de la fermentation et du séchage solaire sur l'acquisition du pouvoir de panification de l'amidon aigre de manioc. In: Transformation Alimentaire du Manioc (eds. T. Agbor Egbe, A. Brauman, G. Griffon \& s. Trèche). Pág. 399-416, Éditions ORSTOM, Montpellier, France.

[FE+99] FERMÁMDEZ A., ZAKHIA M., RUIZ R., TRUJILLO J., 1999. Desarrollo de un método sencillo para medir la calidad del almidón agrio de yuca. Impacto del método sobre la agroindustria rural en el departamento del Cauca (Colombia).

http://www.ciat.cgiar.org/agroempres as/pdf/calidad_almidón.pdf

[LE+98] LEYTOM J., CHARRIA L., 1998; Modelamiento del comportamiento del almidón de yuca en los canales de sedimentación. Proyecto de grado, Plan de Estadística, Universidad del Valle.

[PIN80] PINTO R., 1980. Elaboración y usos del almidón yuca. Boletín técnico Mo. 66. Instituto Colombiano Agropecuario, Bogotá, Colombia.

$[R I+01]$ RIVIER M., MOREMO M., DUFOUR D. 2001. Almidón agrio de yuca en Colombia. Tomo 2: Planta procesadora: descripción y planos de los equipos. Publicación CIAT 323. Cali, Colombia. 
[T+99] TISCHER I., AMDRADE M., MOREMO M., 1999. El mejoramiento de la calidad y la productividad en las rallanderías de yuca. Reporte final de Investigación, Universidad del Valle.

[TKA59] TKATCHEMKO B., 1959. Mote technologique complémentaire pour les féculeries de Madagascar utilisant les bassins de dépôt. Tenanarive, IRAM, pág. 6-37.

[ZA+96] ZAKHIA M., DUFOUR D., CHUZEL G., 1996. Review of sour cassava starch production in rural Colombian areas. Tropical Science 36, 247-255. 